\title{
Efficacy of Philosophical Ethics Uptake in E-learning
}

\author{
Brendan James Moore ${ }^{1, *}$, Syed Adeel Ahmed ${ }^{2}$ \\ ${ }^{1}$ College of Continuing Studies, Tulane University, United States \\ ${ }^{2}$ Department of Medicine-Infectious Diseases, Xavier University of Louisiana, United States
}

Copyright $\subseteq 2016$ by authors, all rights reserved. Authors agree that this article remains permanently open access under the terms of the Creative Commons Attribution License 4.0 International License

\begin{abstract}
E-Learning and Distance Learning has grown as a field over the last twenty years. Business and Universities alike have an interest in being assured that E-Learning can be just as effective as traditional classroom settings. In this paper, seven years of survey data is presented, and analyzed, showing that an analytic, philosophic, ethics courses had noticeable effects on students' who took the course. Students' beliefs about the concepts of rightness and wrongness before taking an online analytic philosophy ethics course noticeably changed after completion of the course, indicating that students are less likely to be Simple Subjectivists after completing an analytic philosophy ethics course.
\end{abstract}

Keywords Philosophy, Professional Ethics, Analytic Philosophy, E-learning, Distance Learning, Learning Objectives, Education

\section{Introduction}

E-Learning and Distance Learning have grown as a field over the last twenty years. Business and Universities alike have an interest in ensuring that Online Education and Online Training can be just as effective as traditional classroom settings. Concerning E-Learning, Ferriman [1] states, "double-digit year over year growth is projected for all regions of the world except Western Europe and North America".

To ensure that learning objectives of courses are met, and due to online education's increasing popularity, research into the effectiveness of teaching online has become even more important now than in the past. One way to test this is through tests, quizzes, discussion boards, and essays. However, there are other indicators of a course's effectiveness. If students honestly report that their stances on deeply held beliefs have changes, then that change may be an indication that the course just taken had been influential.

In order to begin an investigation into this study, since the Distance Learning industry is still relatively new, data had to be collected over the past seven years. After data collection, we will discuss of the results before drawing research conclusions.

\section{Materials and Methods}

\subsection{Materials and Method}

Since 2010, various, mainly non-traditional adult, students, have taken an online introductory ethics course as part of a requirement for an RN-BSN completion program. At Ohio University, 3,454 Survey's were received. The surveys were filled out anonymously and collected using the Blackboard Learning Management System (LMS) survey function.

Before and after an online, introductory ethics courses (medically slanted), students were asked to anonymously submit answers to a survey asking them questions about their various stances on ethics and ethical dilemmas. Since students would submit answers anonymously, there would be less chance of a student purposefully lying to avoid uncalled for judgment from peers or the instructor of record. The surveys were also voluntary filled out insofar as a "Do not Answer" answer was always a selectable option.

\subsection{Hypothesis}

I expected that students would exit the online introductory ethics course with similar results as a traditional classroom setting, meaning that they will be able to achieve the course objectives and possibly have the information that was presented change their previously held stances.

\section{Course Learning Objectives:}

1. State and explain the most influential moral theories in western philosophy as well as the most influential arguments in favor of them and against them.

2. State and explain the central arguments in readings about moral issues prevalent in contemporary American life, especially those that have ramifications for medical ethics.

3. Apply the knowledge and the critical skills to ethical questions that arise in personal, professional, and political situations.

4. Possess the developed skill of participating in a 
philosophical discussion

In addition, according to the University of Kentucky philosophy department [5], studying Analytic Philosophy has also been shown to have the following effects.

1. "analyze, develop and formulate logical arguments

2. capability to make knowledgeable decisions, examining thoroughly the consequences of various actions

3. aptitude to examine various angles of topics

4. ability to write and speak clearly and effectively interpret and assess various thoughts and theories" UK [5].

Given that studying philosophy has the listed above effects on students, I also expected students to generally come out of the class being able to understand debating positions, even debating positions on morally relevant matters, as stemming from the analysis of reasons, rather than merely emoting positions. This might mean that they are more likely to move from a subjectivist stance, to one that relies on things external to their own emotions, such as a general Kantian recognition that the human dignity of another stands apart from their own evaluative judgments, or that the pain and emotions another might feel stands apart from their own, solipsistic, evaluative judgment.

Hypothesis 1: Students will be more likely to derive their moral judgments from reasons and less likely to derive their moral judgments from initial feelings.

Hypothesis 2: Students who are attracted towards ethical theories not supported by the greater philosophic community, will be attracted towards believing the more major theories in ethics.

\subsection{Structure of the Course}

The online introductory ethics courses had two structures. Prior to Summer Semester 2012, the online introductory ethics course was 10 weeks long and structured for a quarter system. After Summer Semester 2012, the online introductory ethics course was structured for 15 weeks and structured for a semester system.

Most of the students were Registered Nurses (RNs) who were continuing education to become BSNs (Bachelor in the Science of Nursing). The course was structured into three sections.

The first section went over some basic points in elementary logic. Students were exposed to the terms argument, premises, and conclusions, as well as the concept of logical validity and logical invalidity. Argument forms such as Modus Ponens, Modus Tollens, Affirming the Consequent, and Denying the Antecedent, were used as examples.

The second section of the course went over basic normative ethical theories. Theories that are by and large rejected by the professional philosophic community include Cultural Relativism/Ethical Relativism, Simple Subjectivism, The Divine Command Theory. Popular "major" ethical theories that are more accepted in the larger philosophic community include Utilitarianism, Kantianism, Aristotelean Virtue Theory, Social Contract Theory, Natural Law Theory.

The third section of the course went over various ethical dilemmas students might face within their industries and how normative ethical theories relate to their profession.

\subsection{What Questions Were Asked}

Questions one and two asked about whether the student supported Cultural Relativism - Rachels [3] - and what Lent [2] calls Ethical Relativism. Rachels [3] gives a lengthy set of claims for Cultural Relativists:

"1. Different societies have different moral codes.

2. The moral code of a society determines what is right within that society: that is, if the moral code of a society says that a certain action is right, then that action is right, at least within that society.

3. There is no objective standard that can be used to judge one society's code as better than another's. There are no moral truths that hold for all people at all times.

4. The moral code of our own society has no special status; it is but one among many.

5. It is arrogant for us to judge other cultures. We should always be tolerant of them." Rachels [3].

So, if Ethical Relativism were true, according to Rachels [3]:

1. "We could no longer say that the customs of other societies are morally inferior to our own" (ex: we cannot say that oppressive societies are worse than non-oppressive).

2. "We could no longer criticize the code of our own society" (seems to just affirm the way things currently are: things could never be said to change for the better)

3. "The idea of moral progress is called into doubt" (social reforms could not be seen as correct or justified)

Question 3 identifies students' stance towards Simple Subjectivism.

Simple Subjectivism: Simple Subjectivism is the stance that when we use terms such as right and wrong, they are only referring to what we feel. Whether we feel an action is right or wrong makes that action right or wrong.

Simple Subjectivism is not generally understood as an objective stance in ethics, since the mind-dependent and first-person nature of the stance makes subjective the rightness and wrongness of actions.

Question 4 identifies students' stance towards Psychological Egoism.

Psychological Egoism: the descriptive claim that when people act, they always do so towards their self-interest, even if that is not a motivating feature of their conscious awareness.

Question 8 identifies students' stance towards Utiltiarianism.

Utilitarianism: Rachels [3] defines Bentham's Classical Utiltiarian system as including three features.

1. Consequensalist: consequenses are the only thing evaluated in determining whether or not an action is right or wrong 
2. Hedonism: The maximization of pleasure and the minimizing of pain are the only morally relevant considerations

3. Impartiality: Every person's (or sentient being's) suffering matters equally.

Question 14 identifies students' stance towards Ethical Egoism

Ethical Egoism: Ethical Egoism is the stance that one ought to act in their self-interest, understood as their best-interest

Question 15 identifies whether or not the student has an absolutist view about ethics, and question 6 identifies one way in which a student might be a deontologist.

Figure 1, question one, asks, "Morality differs in every society, and is nothing more than a convenient term for socially approved habits.", with five options: Strongly Agree, Agree, Neither Agree nor Disagree, Disagree, and Strongly Disagree.

For Question 1, when students completed the preliminary survey, 136 selected strongly agree, 843 selected agree, 184 selected neither agreed nor disagreed, 555 selected disagree, and 58 selected strongly disagree. When students completed the post ethics course survey, 213 selected strongly agree, 713 selected agree, 94 selected neither agree nor disagree, 478 selected disagree, and 78 selected strongly disagree.

If the number 1 represented Strongly Agree and 5 represented Strongly Disagree, the mean score for the pre-course survey is 2.75 and the mean for the post-course survey is 2.67 .

\subsection{Figures and Results}

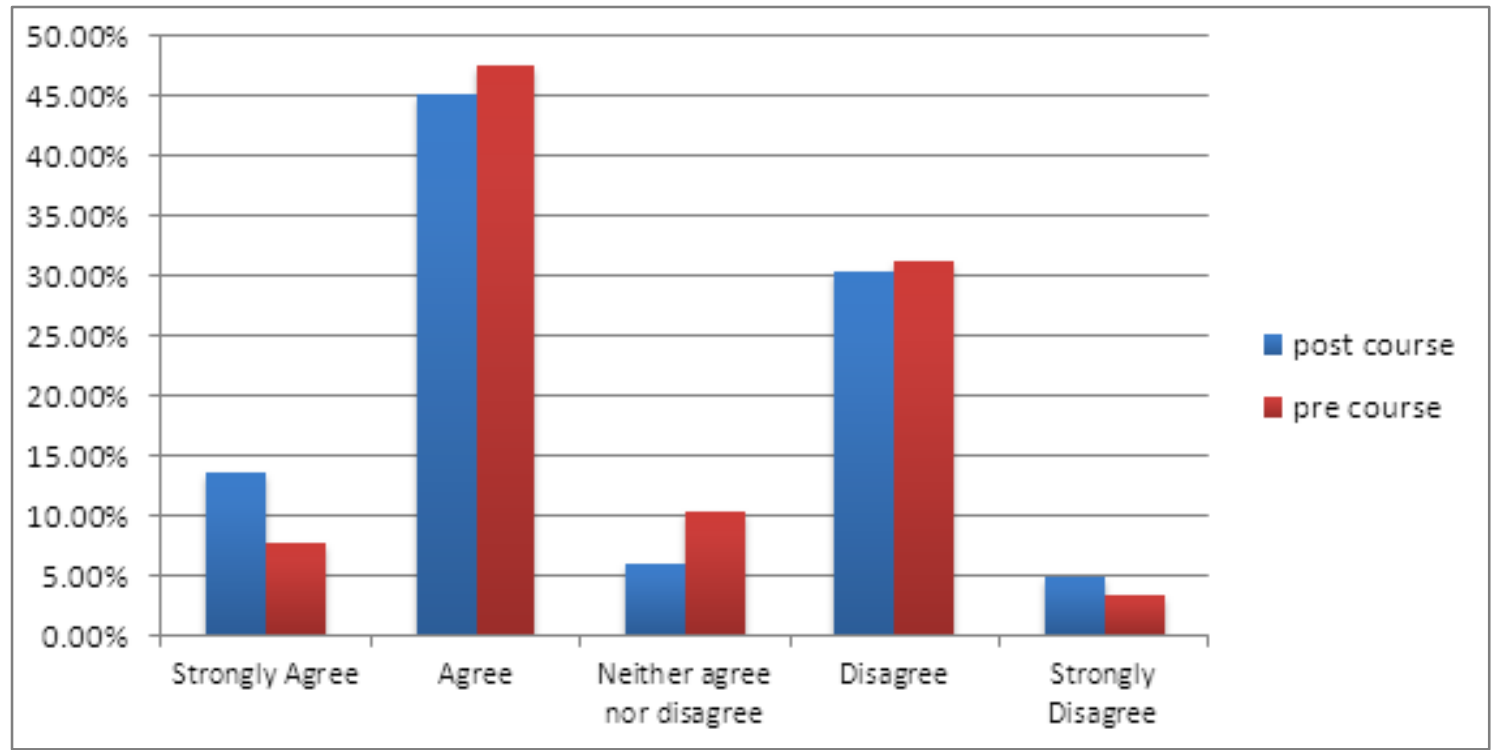

Figure 1. Question 1, on Cultural Relativism

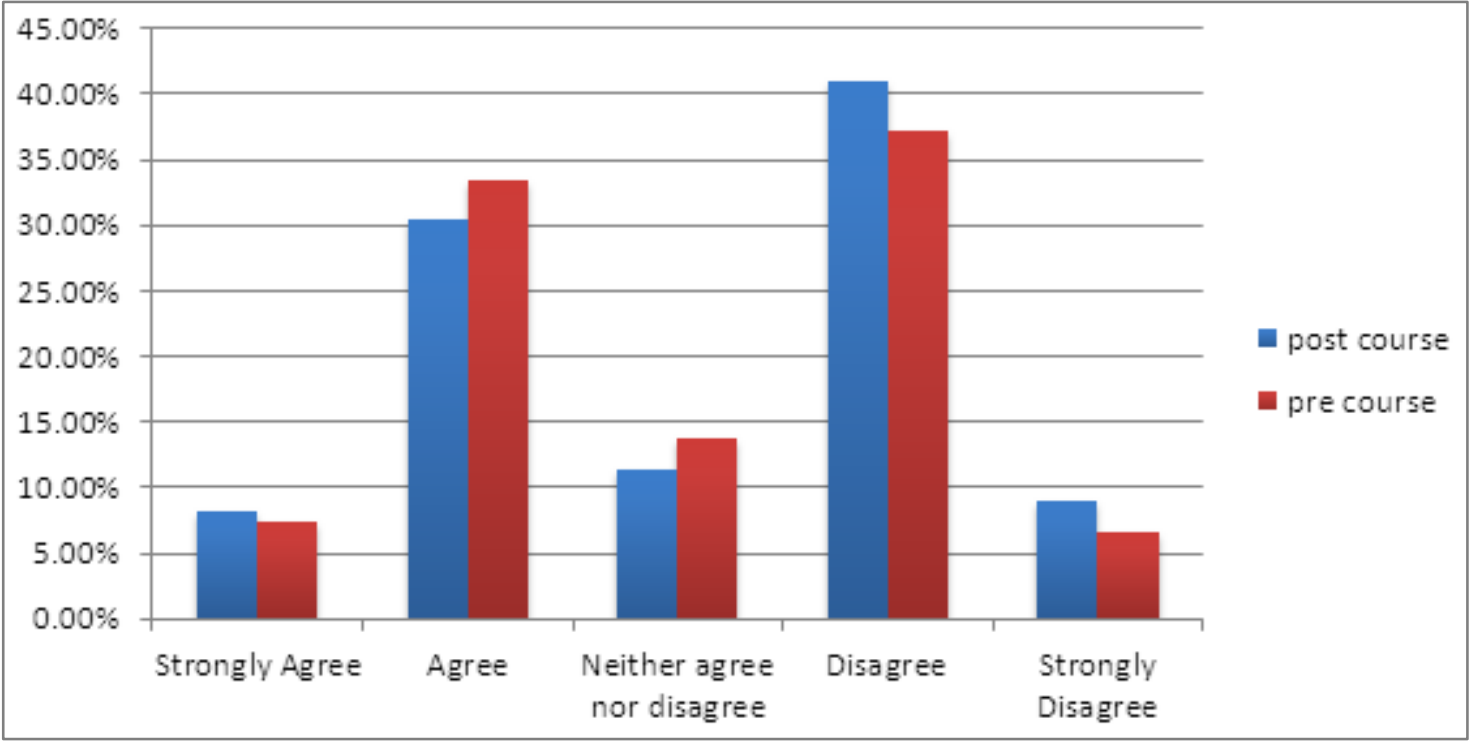

Figure 2. Question 2, on Cultural Relativism 
Figure 2, question two, asks, "The best guide to what is morally right and morally wrong is what one's culture believes is right.", with five options: Strongly Agree, Agree, Neither Agree nor Disagree, Disagree, and Strongly Disagree.

For Question 2, when students completed the preliminary survey, 133 selected strongly agree, 606 selected agree, 257 selected neither agreed nor disagreed, 661 selected disagree, and 116 selected strongly disagree. When students completed the post ethics course survey, 128 selected strongly agree, 479 selected agree, 181 selected neither agree nor disagree, 646 selected disagree, and 142 selected strongly disagree.

If the number 1 represented Strongly Agree and 5 represented Strongly Disagree, the mean score for the pre-course survey is 3.01 and the mean for the post-course survey is 3.12 .

Figure 3, question three, asks, "The best guide to what is morally right and wrong is what my own feelings tell me.", with five options: Strongly Agree, Agree, Neither Agree nor Disagree, Disagree, and Strongly Disagree.

For Question 3, when students completed the preliminary survey, 358 selected strongly agree, 764 selected agree, 252 selected neither agreed nor disagreed, 344 selected disagree, and 59 selected strongly disagree. When students completed the post ethics course survey, 179 selected strongly agree, 365 selected agree, 194 selected neither agree nor disagree, 593 selected disagree, and 188 selected strongly disagree.

If the number 1 represented Strongly Agree and 5 represented Strongly Disagree, the mean score for the pre-course survey is 2.43 and the mean for the post-course survey is 3.16 .

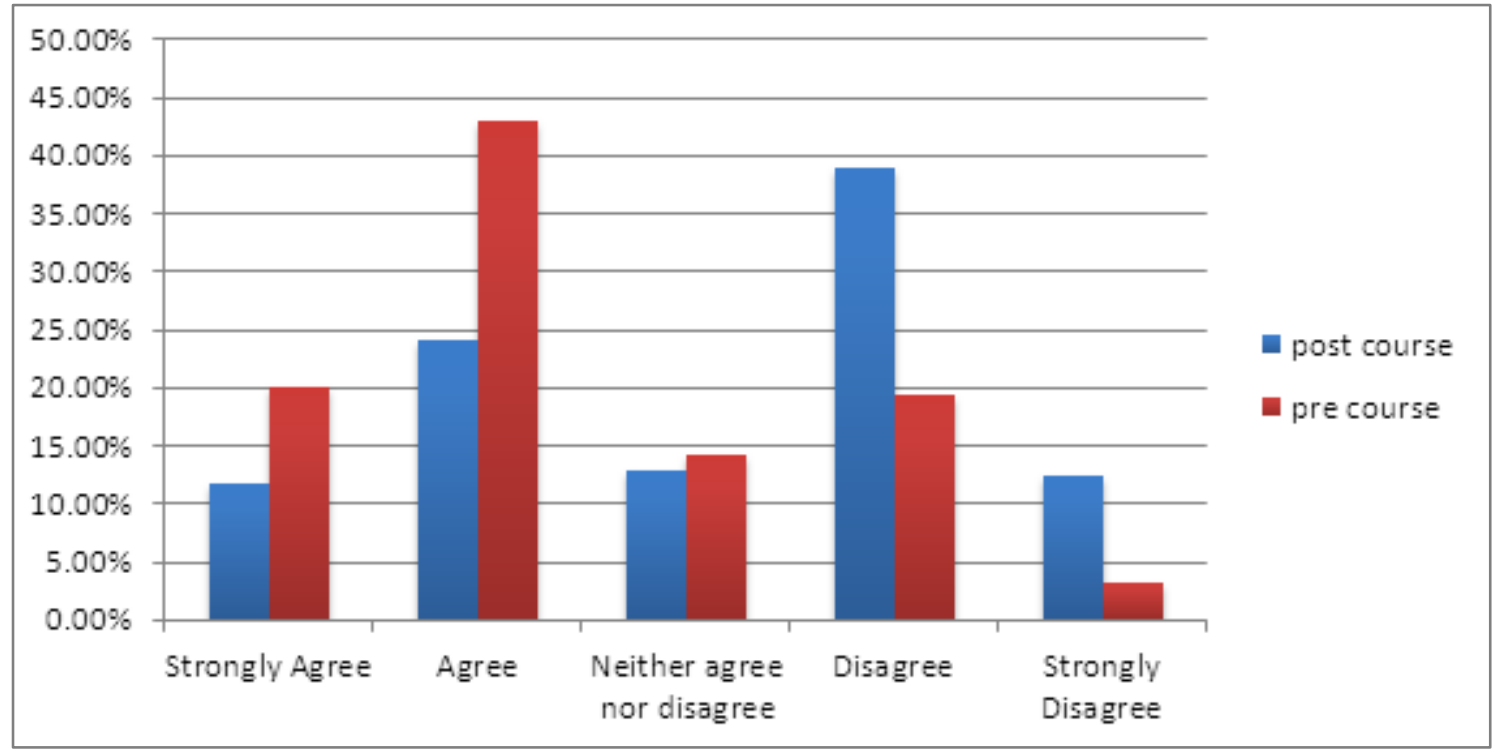

Figure 3. Question 3 on Simple Subjectivism

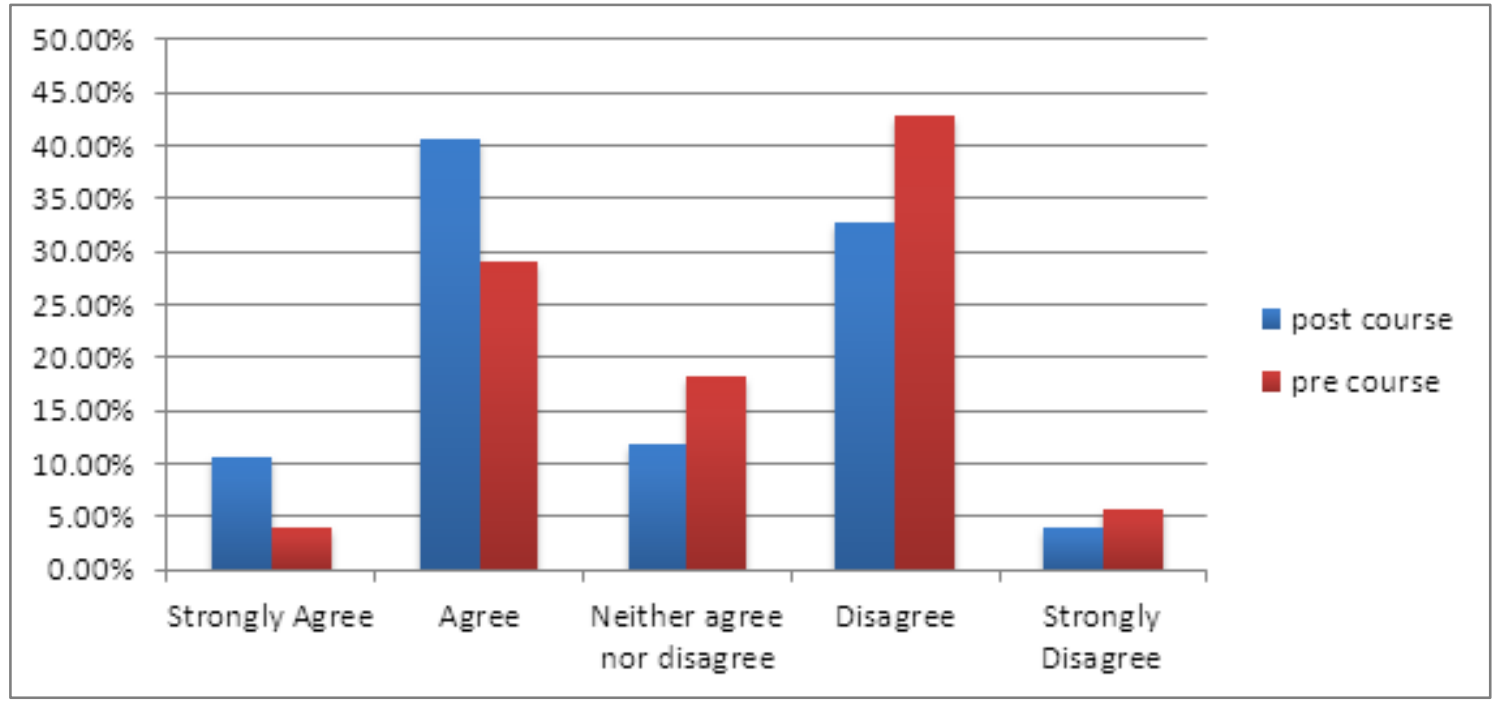

Figure 4. Question 4, on Psychological Egoism 
Figure Four, question four, asks, "Even when a person appears to be making a sacrifice for another person, the aim is always some good for the person doing the action", with five options: Strongly Agree, Agree, Neither Agree nor Disagree, Disagree, and Strongly Disagree.

For Question 4, when students completed the preliminary survey, 70 selected strongly agree, 516 selected agree, 326 selected neither agreed nor disagreed, 763 selected disagree, and 102 selected strongly disagree. When students completed the post ethics course survey, 168 selected strongly agree, 639 selected agree, 188 selected neither agree nor disagree, 515 selected disagree, and 65 selected strongly disagree.

If the number 1 represented Strongly Agree and 5 represented Strongly Disagree, the mean score for the pre-course survey is 3.18 and the mean for the post-course survey is 2.79 .

Figure 5, question six, asks, "It is always wrong to take a human life "with five options: Strongly Agree, Agree, Neither Agree nor Disagree, Disagree, and Strongly Disagree.

For Question 6, when students completed the preliminary survey, 421 selected strongly agree, 416 selected agree, 326 selected neither agreed nor disagreed, 516 selected disagree, and 97 selected strongly disagree. When students completed the post ethics course survey, 146 selected strongly agree, 266 selected agree, 201 selected neither agree nor disagree, 686 selected disagree, and 276 selected strongly disagree.

If the number 1 represented Strongly Agree and 5 represented Strongly Disagree, the mean score for the pre-course survey is 2.69 and the mean for the post-course survey is 3.43 .

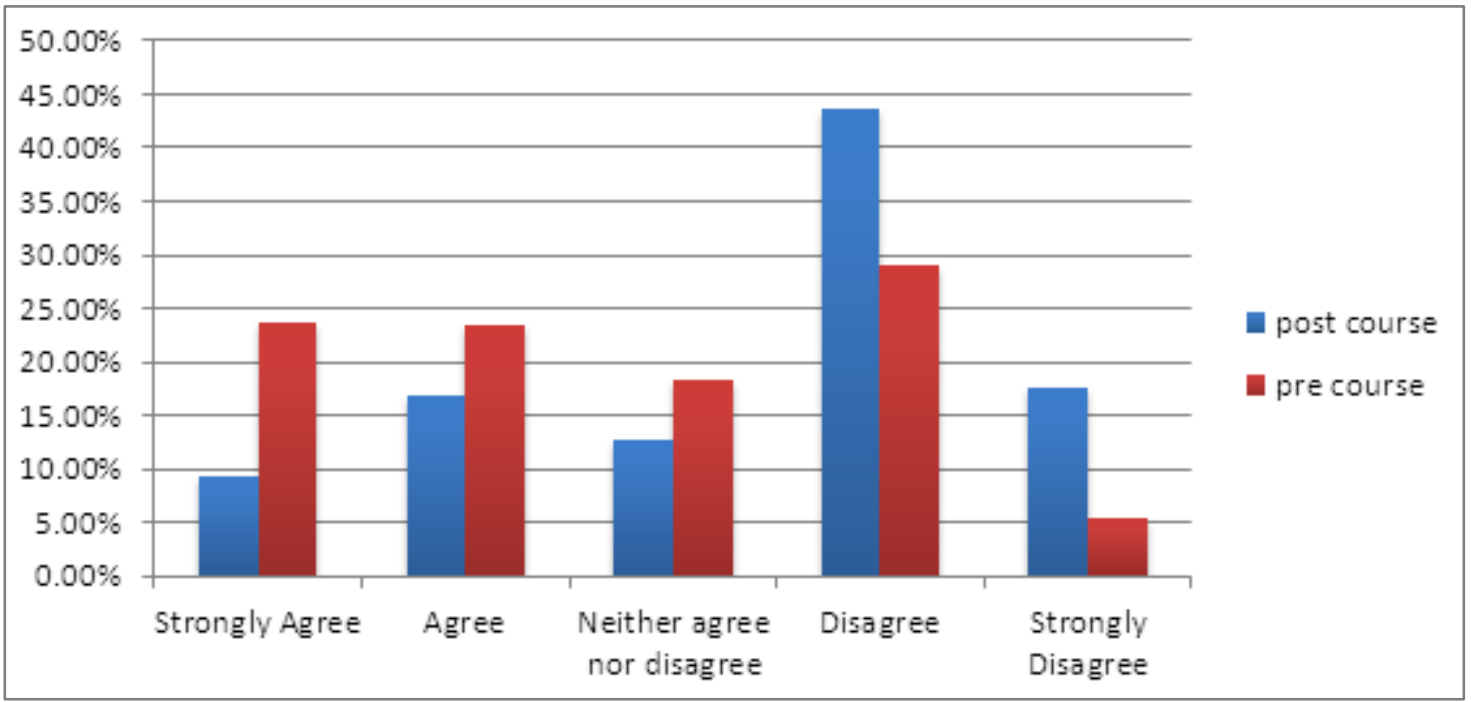

Figure 5. Question 6 on the wrongness of killing another human

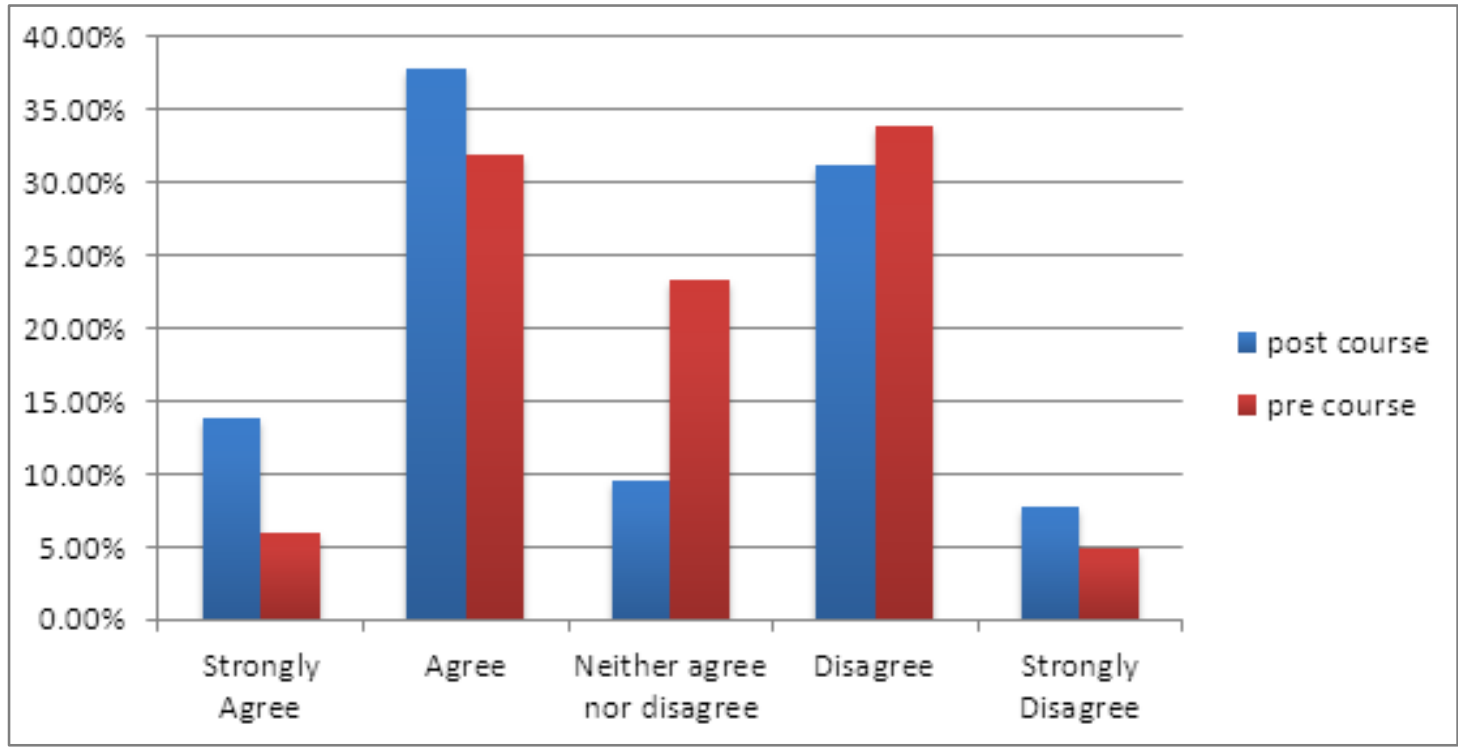

Figure 6. Question 8 on Utilitarianism 
Figure 6, question eight, asks, "If a human being can suffer, there is no moral justification for refusing to take that suffering into consideration" with five options: Strongly Agree, Agree, Neither Agree nor Disagree, Disagree, and Strongly Disagree.

For Question 8, when students completed the preliminary survey, 106 selected strongly agree, 567 selected agree, 413 selected neither agreed nor disagreed, 600 selected disagree, and 88 selected strongly disagree. When students completed the post ethics course survey, 218 selected strongly agree, 594 selected agree, 150 selected neither agree nor disagree, 490 selected disagree, and 122 selected strongly disagree.

If the number 1 represented Strongly Agree and 5 represented Strongly Disagree, the mean score for the pre-course survey is 3.00 and the mean for the post-course survey is 2.81 .

Figure 7, question fourteen, asks, "Morality is just a convenient term for self-interest" with five options: Strongly Agree, Agree, Neither Agree nor Disagree, Disagree, and Strongly Disagree.

For Question 14, when students completed the preliminary survey, 280 selected strongly agree, 281 selected agree, 321 selected neither agreed nor disagreed, 750 selected disagree, and 143 selected strongly disagree. When students completed the post ethics course survey, 202 selected strongly agree, 310 selected agree, 226 selected neither agree nor disagree, 679 selected disagree, and 158 selected strongly disagree.

If the number 1 represented Strongly Agree and 5 represented Strongly Disagree, the mean score for the pre-course survey is 3.11 and the mean for the post-course survey is 3.18 .

Figure 8, question fifteen, asks, "Some things are just wrong and may not be done, even if good may come from doing them" with five options: Strongly Agree, Agree, Neither Agree nor Disagree, Disagree, and Strongly Disagree.

For Question 15, when students completed the preliminary survey, 93 selected strongly agree, 535 selected agree, 388 selected neither agreed nor disagreed, 672 selected disagree, and 97 selected strongly disagree. When students completed the post ethics course survey, 167 selected strongly agree, 560 selected agree, 212 selected neither agree nor disagree, 548 selected disagree, and 89 selected strongly disagree.

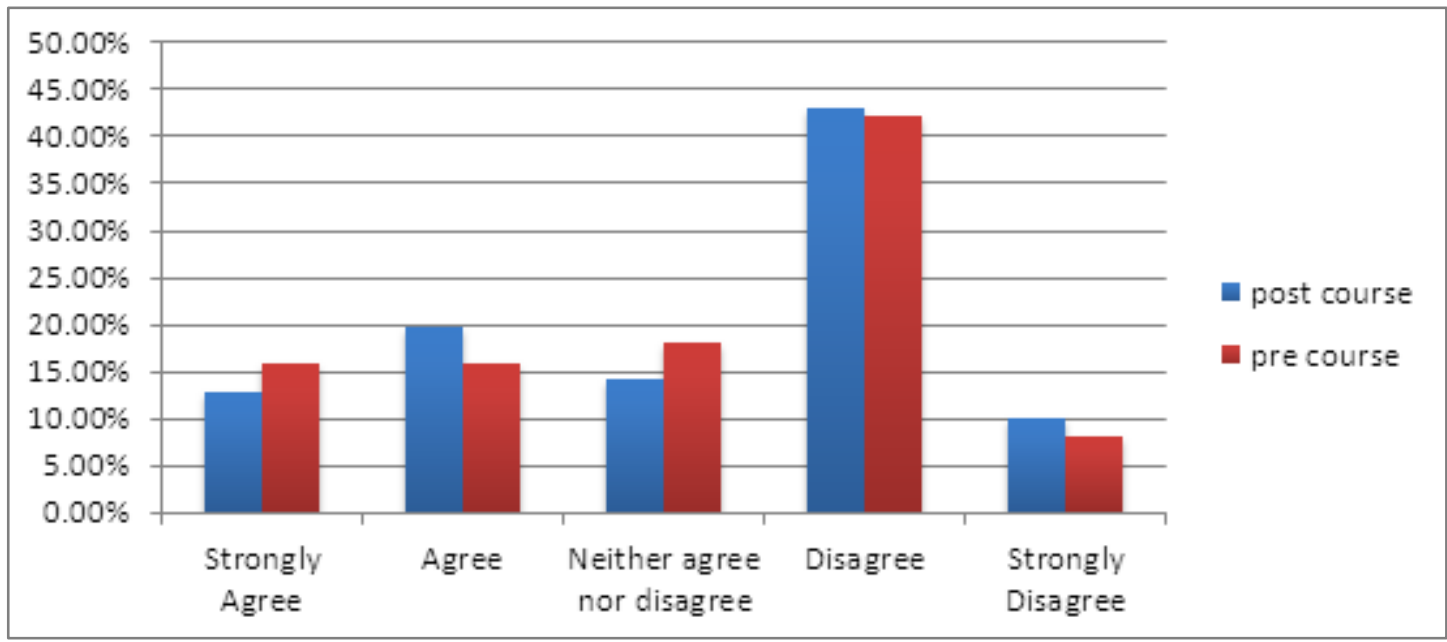

Figure 7. Question 14 on Ethical Egoism

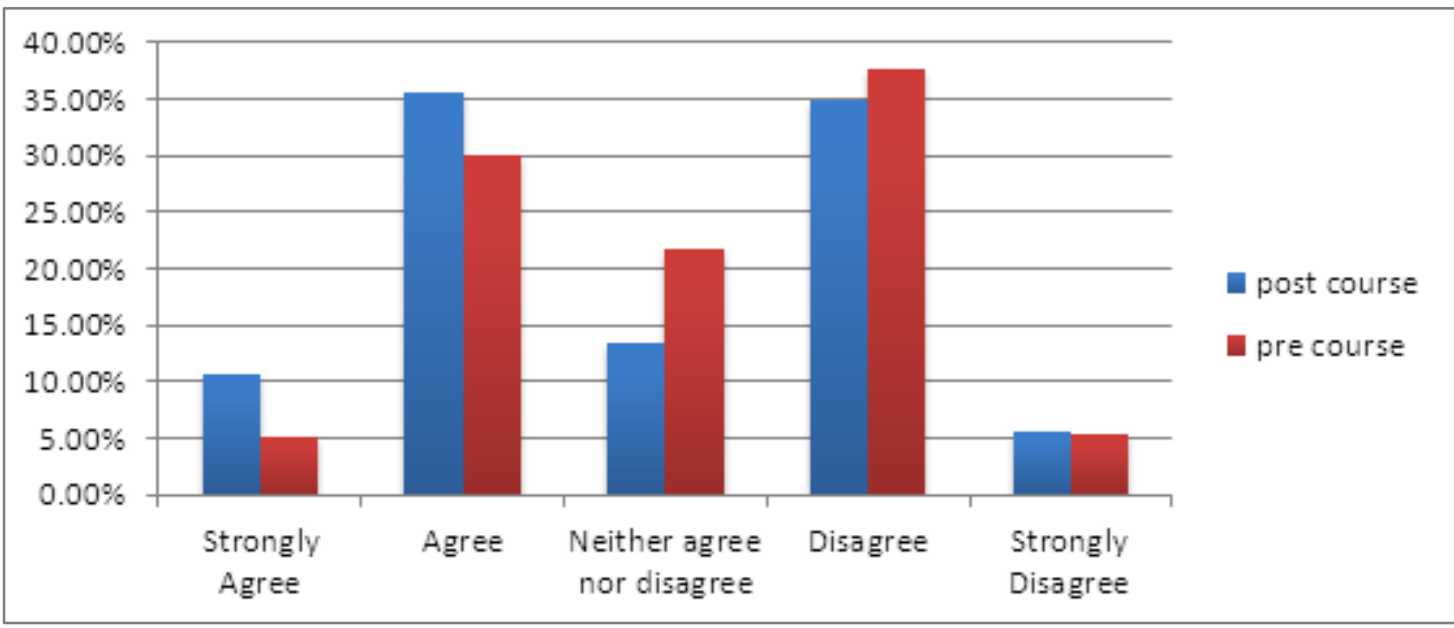

Figure 8. Question 15, on absolutism in Ethics 
If the number 1 represented Strongly Agree and 5 represented Strongly Disagree, the mean score for the pre-course survey is 3.08 and the mean for the post-course survey is 2.89 .

\subsection{Discussion}

There are several things to notice. My first hypothesis was correct. Figure 3 indicates that many students changed from a Simple Subjectivist stance (the view that what is morally right and wrong is simply shorthand for their own feelings) to one where they reject that their feelings are the best guide to understanding what's right and wrong.

One thing that surprised me and was unexpected is that more people became psychological egoists (that appearances of self-sacrifice are always, at bottom, rooted in self-interest. Even though Ayn Rand understood Ethical Egoism (the view that we ought to act in our self-interest understood as our best-interest) as an objective theory in ethics, the view is still widely regarded by professional philosophers as failing compared to other major theories (Utilitarianism, Kantianism, Aristotelean Virtue Theory, Social Contract Theory).

Although Figure 7 supports that students held Ethical Egoist dispositions throughout the course, Figure 6 contradicts it insofar as the suffering of sentient beings ought to always be taken into account contradicts the unfettered per suit of self-interest.

Figures 1 and 2 also led to some surprising results. Even though the cohort's answers did not change between the beginning and the end of the course, I was surprised to see that the majority of students disagreed with question 2 , while the majority of students agreed with question 1 , even though both are entailments of Cultural Relativism. There might be cognitive dissonance (the holding of two mutually incompatible propositions as true at the same moment) among some students, but there may be another explanation, which will be elaborated in the "Limitations" section of this paper.

When a student makes a judgment about Morality, or what is "morally right", they might not intend the judgment of something being morally right as implying that the action would be "the best thing to do". Nor would someone necessarily have to accept that knowing the morally best thing to do would end up being the best guide to how they ought to act. If this reasoning were the case, questions 1 and 2 would not entail one another and there would not be the cognitive dissonance conjectured earlier.

My first hypothesis was correct. However, my second hypothesis was incorrect.

Students also were more likely to be Ethical Egoists after the course and about as likely to support Utilitarian principles as prior to the course. They were also far less likely to be Simple Subjectivists after having taken the course.

On a side note, students were far less likely to view the taking of a human life as always morally wrong.

\section{Limitations}

The first limitation to notice is that the wording of the questions may be ambiguous. When someone reads a question about God, for example, they may have their own conception in use, rather than the one that would best suit the underlying theory in ethics the question is trying to assess. For example, the Divine Command Theory and Aquinas' Theory of Natural Law use differing conceptions of God, and depending on which one is in use, the student may answer the survey question differently. Similarly, students were not primed before answering questions.

If a student had Self-Defense on their mind, as opposed to Capital Punishment, they may have answered question six (figure 5).

The second limitation to notice is the population. The students being tested, although all online, are still enrolled in a mid-sized university in the mid-west, which might attract a certain demographic of the population that leads to results that are not generalizable to teaching methods in other areas.

Also, since most of the students were RNs working on their BSNs, the results may not be generalizable to the general population.

A third limitation is that simply because we know that the percentage of students before and after the course stayed the same, does not mean that we can conclude that individual students' stances were unchanged. Many students may have changed their stance, yet the overall percentage may not reflect the amount of change.

\subsection{Appendix}

\section{Listed here is the original Survey}

1. Morality differs in every society, and is nothing more than a convenient term for socially approved habits.

2. The best guide to what is morally right and morally wrong is what one's culture believes is right.

3 . The best guide to what is morally right and wrong is what my own feelings tell me.

4. Even when a person appears to be making a sacrifice for another person, the aim is always some good for the person doing the action.

5. There is no difference between actually killing a person and knowingly letting that person die.

6. It is always wrong to take a human life.

7. Taking a human life is the same thing morally as doing nothing when it is in your power to save a life.

8. If a human being can suffer, there is no moral justification for refusing to take that suffering into consideration.

9. If God commanded us to torture children for sport (assuming God exists), it would be morally right to do so and morally wrong not to do so.

10. A person with a terminal disease whose death grows near and increasingly painful and who wants to commit suicide should be able to do so without interference.

11. A person with a terminal disease whose death grows 
near and increasingly painful and who wants to commit suicide should be able to receive the necessary help from healthcare professionals.

12. A woman has the right to do whatever she chooses with her body.

13. A fetus is a person from the moment of conception.

14. Morality is just a convenient term for self-interest.

15. Some things are just wrong and may not be done, even if good may come from doing them.

16. I know what I believe about what is right and what is wrong, and I believe that I have good reasons for holding the beliefs I do.

17. Experimenting on nonhuman animals is justified if humans will benefit.

18. A man has a right to do whatever he chooses to do with his body.

19. My ethical beliefs are the result of careful deliberation.

20 . Some of my deeply held moral beliefs might be wrong.

21 . When two people have a disagreement about an ethical issue, nothing can be gained by arguing over it.

22. Everyone is entitled to his or her opinion on ethical matters. All opinions on ethical matters are equally valid.

\section{Listed here is an interpretation of what the questions are asking}

1: Do you support cultural relativism?

2. Do you support cultural relativism?

3. Do you support simple subjectivism?

4. Do you support psychological egoism?

5. Do you support the notion that there is no distinction between active and passive euthanasia?

6. Do you support the notion that killing a human life is always wrong?

7. Do you support that actively killing a human is the same morally as passive killing a human (when something can be done to intervene)?

8. Do you support that the suffering of sentient beings is always a morally relevant consideration?

9. Do you support that if God exists, he can command in an unconstrained way what is morally right and morally wrong?

10. Do you support someone committing suicide (in the case of a terminally ill patient)?

11. Do you support physician-assisted suicide (in the case of a terminally ill patient)?

12. Do you support that a woman may do what she wants with her body?

13. Is the fetus a person?

14. Do you support Ethical Egoism?

15. Do you support Objectivism in ethics, even aside from pragmatic concerns?

16. Do you support that you have good reasons for holding your beliefs?

17. Do you support Speciesism?

18. Do you support that a man may do what he wants with his body?
19. Do you support that your ethical beliefs are the result of careful deliberation?

20. Do you support that some of your moral beliefs may be wrong?

21. Do you support that argument over ethical issues is pointless?

22. Do you support ethical subjectivism?

\section{Conclusions}

In conclusion, students were far less likely to be Simple Subjectivists by the end of the analytic online introductory ethics course and more likely to support Ethical Egoism, although their support of Cultural Relativistic principles and Utiltiarian principles were less likely to have changed.

More research is needed in the area of the effectiveness of online education, and more work should be done before drawing any definite conclusions about the efficacy of online analytic philosophy ethics courses.

In future research, comparing the same course with the same structure in the classroom setting to one in an online format would be more useful, since departments and courses vary in the material they teach across regions of the world. Also, rather than comparing group shifts, looking at whether results on individual surveys changed would give better indication that the course is changing any particular individual's position.

\section{Acknowledgements}

Thank you Dr. Alfred C. Lent, and Ohio University's philosophy department for guidance and giving me the opportunity to teach online for the last 7 years. Also, thanks to Dr. Syed Adeel Ahmed, Tulane University for guidance and mentoring through the Business of Information Technology course in the Masters of Professional Studies program for Computing Technology.

\section{REFERENCES}

[1] Ferriman, Justin, "The Explosive Growth of ELearning", Learning and Collaboration Blog, LearndDash, September 17, 2014

[2] Lent, Alfred C., The Building Project: a novel about a workaday philosopher, 2010.. https://www.amazon.com/Buil ding-Project-novel-workaday-philosopher-ebook/dp/B006O P2ZBC

[3] Rachels, James, The Elements of Moral Philosophy $7^{\text {th }}$ edition edited by Stuart Rachels, McGraw Hill, 2010.

[4] Rachels, James, The Right Thing to Do $6^{\text {th }}$ edition edited by Stuart Rachels, McGraw Hill, 2010.

[5] University of Kentucky, "Where Can Philosophy Take Me?", College of Arts and Sciences, 2016. https://philosophy.as.uk y.edu/where-can-philosophy-take-me

[6] Williams, Brian K., Sawyer, Stacey K., Berston, Susan, Business: A Practical Introduction, $1^{\text {st }}$ Edition, Pearson 2014. 